\title{
PRODUCT SHIFTS ON $B(H)$
}

\author{
P. J. STACEY
}

(Communicated by Palle E. T. Jorgensen)

\begin{abstract}
A shift on $B(H)$ is a *-endomorphism $\alpha$ for which $\bigcap_{r} \alpha^{r}(B(H))=$ $\mathbb{C} P$ for some projection $P$. The paper discusses some aspects of the classification of shifts on $B(H)$ up to conjugacy by *automorphisms, with a focus on the shifts arising from an infinite tensor product decomposition of $H$.
\end{abstract}

\section{INTRODUCTION}

Let $H$ be a separable Hilbert space and let $B(H)$ be the algebra of all bounded linear operators on $H$. Slightly extending the definition given in [7] to include the nonunital case, a *-endomorphism $\alpha$ of $B(H)$ will be called a shift if $\bigcap_{r} \alpha^{r}(B(H))=\mathbb{C} P$ for some (possibly zero) projection $P$ on $B(H)$. The present paper discusses some aspects of the classification of shifts on $B(H)$ up to conjugacy by *-automorphisms, with a focus on the shifts arising from an infinite tensor product decomposition of $H$.

\section{SPATIAL DESCRIPTIONS}

It was observed in [2, Proposition 2.1] that every nonzero normal *-endomorphism $\alpha$ of $B(H)$ is implemented by a sequence of isometries. An alternative proof of this result can be obtained by imitating the usual proof that *automorphisms of $B(H)$ are inner, as described, for example, in [5, Lemma 7.5.3], and this alternative proof shows that the normality assumption can be omitted (and hence every *-endomorphism of $B(H)$ is normal). For later reference we now state this minor extension of [2, Proposition 2.1].

Proposition 2.1. Let $\alpha$ be a nonzero *-endomorphism of $B(H)$. Then there is a (finite or infinite) sequence of isometries $V_{1}, V_{2}, \ldots$ in $B(H)$ having mutually. orthogonal ranges such that

$$
\alpha(A)=\sum_{n} V_{n} A V_{n}^{*}
$$

Received by the editors May 7, 1990.

1980 Mathematics Subject Classification (1985 Revision). Primary 46L40; Secondary 46L55, $47 \mathrm{~A} 65$.

Key words and phrases. Endomorphism, isometry, Hilbert space, shift, infinite tensor product, unitary equivalence. 
for each $A \in B(H)$. The linear space of operators

$$
E_{\alpha}=\{T \in B(H): \alpha(A) T=T A \text { for all } A \in B(H)\}
$$

is a Hilbert space relative to the inner product defined by

$$
T^{*} S=\langle S, T\rangle 1
$$

and $\left\{V_{1}, V_{2}, \ldots\right\}$ is an orthonormal basis for $E_{\alpha}$.

The following simple result relates the conjugacy of $\alpha$ and $\beta$ to the Hilbert spaces $E_{\alpha}$ and $E_{\beta}$.

Proposition 2.2. Let $\alpha, \beta$ be *endomorphisms of $B(H)$ and let $U \in B(H)$ be unitary. Then $\alpha=(\operatorname{Ad} U) \beta\left(\operatorname{Ad} U^{*}\right)$ if and only if $E_{\alpha}=U E_{\beta} U^{*}$.

Proof. If $\alpha=(\operatorname{Ad} U) \beta\left(\operatorname{Ad} U^{*}\right)$ then an easy calculation yields $E_{\alpha}=U E_{\beta} U^{*}$. This same result shows that if $E_{\alpha}=U E_{\beta} U^{*}$ then $E_{\alpha}=E_{\gamma}$ where $\gamma=$ $(\operatorname{Ad} U) \beta\left(\operatorname{Ad} U^{*}\right):$ hence there exist orthonormal bases $\left\{V_{1}, V_{2}, \ldots\right\}$ and $\left\{W_{1}, W_{2}, \ldots\right\}$ of $E_{\alpha}$ with $\alpha(A)=\sum V_{n} A V_{n}^{*}$ and $\gamma(A)=\sum W_{n} A W_{n}^{*}$ for each $A \in B(H)$. A simple calculation using the unitary transition matrix between the two orthonormal bases then yields the conclusion $\alpha=\gamma$.

A natural question arising from Propositions 2.1 and 2.2 is the extent to which the specification of the isometries $V_{i}$ up to unitary equivalence determines the corresponding *-endomorphism up to conjugacy. The classification of an isometry $V$ up to unitary equivalence is given by the Wold decomposition, described, for example, in [4, Problem 118], which decomposes $V$ as a direct sum of copies of the unilateral shift and a unitary mapping on a subspace; when the unitary summand is absent the isometry is said to be pure. It will now be shown that if $\alpha$ is a shift, then at most one of any corresponding family of isometries is not pure; this result extends [1, Theorem 3] which is the case of two unitarily equivalent isometries. The proof is based on the following lemma in which Cuntz's notation $V_{\mu} V_{\nu}^{*}$ is used to denote $V_{\mu(1)} \cdots V_{\mu(r)} V_{\nu(s)}^{*} \cdots V_{\nu(1)}^{*}$, where $\mu=(\mu(1), \ldots, \mu(r))$ has $r=|\mu|$ components and $\nu=(\nu(1), \ldots, \nu(s))$ has $s=|\nu|$ components.

Lemma 2.3. Let $V_{1}, V_{2}, \ldots$ be isometries from a Hilbert space $H$ onto a family of mutually orthogonal subspaces; $\alpha$ be the *-endomorphism defined by $\alpha(A)=$ $\sum_{n} V_{n} A V_{n}^{*} ;$ and $h \in \bigcap_{r} V_{i}^{r} H$.

(i) If $P_{h}$ is the orthogonal projection from $H$ onto the closed linear span $S$ of the vectors $V_{\mu} V_{\nu}^{*} h$ with $|\mu| \geq 0$ and $|\nu| \geq 0$, then $\alpha\left(P_{h}\right)=P_{h}$ and hence $P_{h} \in \bigcap_{r} \alpha^{r}(B(H))$.

(ii) If $Q_{h}$ is the orthogonal projection from $H$ onto the closed linear span $T$ of the vectors $V_{\mu} V_{\nu}^{*} h$ with $|\mu|=|\nu| \geq 0$, then $Q_{h} \in \bigcap_{r} \alpha^{r}(B(H))$.

Proof. (i) Let $|\mu|=r,|\nu|=s$, and $h=V_{i}^{s+1} k$, where $k \in H$. Then $\alpha(1) V_{\mu} V_{\nu}^{*} h=\sum V_{n} V_{n}^{*} V_{\mu} V_{\nu}^{*} V_{i}^{s+1} k=V_{\mu} V_{\nu}^{*} h$ (even if $r=0$ ) and hence $\alpha(1) \geq$ $P_{h}$. Since $S$ is invariant under both $V_{n}$ and $V_{n}^{*}$, it follows that $P_{h} V_{n}=V_{n} P_{h}$ 
for each $n$ and hence $\alpha\left(P_{h}\right)=\sum V_{n} P_{h} V_{n}^{*}=P_{h} \sum V_{n} V_{n}^{*}=P_{h} \alpha(1)=P_{h}$.

(ii) Since $T$ is invariant under each $V_{\gamma} V_{\delta}^{*}$ with $|\gamma|=|\delta|$, it follows that $Q_{h} V_{\gamma} V_{\delta}^{*}=V_{\gamma} V_{\delta}^{*} Q_{h}$. Thus

$$
\begin{aligned}
\alpha^{r}\left(V_{1}^{* r} Q_{h} V_{1}^{r}\right) & =\sum_{|\gamma|=r} V_{\gamma} V_{1}^{* r} Q_{h} V_{1}^{r} V_{\gamma}^{*} \\
& =\sum_{|\gamma|=r} V_{\gamma} V_{1}^{* r} V_{1}^{r} V_{\gamma}^{*} Q_{h}=\sum_{|\gamma|=r} V_{\gamma} V_{\gamma}^{*} Q_{h} \\
& =\alpha^{r}(1) Q_{h} .
\end{aligned}
$$

However, using part (i), $Q_{h} \leq P_{h}=\alpha^{r}\left(P_{h}\right) \leq \alpha^{r}(1)$ so $Q_{h}=\alpha^{r}\left(V_{1}^{* r} Q_{h} V_{1}^{r}\right)$ and hence $Q_{h} \in \bigcap_{r} \alpha^{r}(B(H))$.

Proposition 2.4. Let $V_{1}, V_{2}, \ldots$ be a sequence of isometries from a Hilbert space $H$ onto a family of orthogonal subspaces and let $\alpha$ be the *-endomorphism of $B(H)$ defined by $\alpha(A)=\sum_{n} V_{n} A V_{n}^{*}$. If $\alpha$ is a shift, then at most one of the isometries $V_{n}$ is not pure and if $V_{n}$ is not pure then the subspace $\bigcap_{r} V_{n}^{r} H$ associated with the unitary summand of $V_{n}$ is one-dimensional.

Proof. Suppose, to obtain a contradiction, that there exist nonzero vectors $h \in \bigcap_{r} V_{i}^{r} H$ and $k \in \bigcap_{r} V_{j}^{r} H$ with $\langle h, k\rangle=0$. Then, by part (ii) of the lemma, there exist associated nonzero projections $Q_{h}, Q_{k}$ in $\bigcap_{r} \alpha^{r}(B(H))$. Let $|\mu|=|\nu|$ and $|\delta|=|\gamma|$. The proof will be completed if it is shown that $\left\langle V_{\mu} V_{\nu}^{*} h, V_{\gamma} V_{\delta}^{*} k\right\rangle=0$ for then $Q_{h} Q_{k}=0$ and, therefore, $\alpha$ is not a shift. To this end let $V_{\delta} V_{\gamma}^{*} V_{\mu} V_{\nu}^{*}=V_{\rho} V_{\sigma}^{*} ;|\rho|=|\sigma|=r ; h=V_{i}^{r+1} h^{\prime}$; and $k=V_{j}^{r+1} k^{\prime}$. Then $\left\langle V_{\mu} V_{\nu}^{*} h, V_{\gamma} V_{\delta}^{*} k\right\rangle=\left\langle V_{\rho} V_{\sigma}^{*} h, k\right\rangle=\left\langle V_{\sigma}^{*} h, V_{\rho}^{*} k\right\rangle=$ $\delta_{\sigma(i, \ldots, i)} \delta_{\rho(j, \ldots, j)}\left\langle V_{i} h^{\prime}, V_{j} k^{\prime}\right\rangle$. But, when $i \neq j,\left\langle V_{i} h^{\prime}, V_{j} k^{\prime}\right\rangle=\left\langle h^{\prime}, V_{i}^{*} V_{j} k^{\prime}\right\rangle$ $=0$ and, when $i=j,\left\langle V_{i} h^{\prime}, V_{j} k^{\prime}\right\rangle=\left\langle V_{i}^{* r} h, V_{i} k^{\prime}\right\rangle=\left\langle h, V_{i}^{r+1} k^{\prime}\right\rangle=\langle h, k\rangle=0$, as required.

Using Proposition 2.4, it is possible to give a complete classification up to conjugacy of the simplest shifts $\alpha$, for which $E_{\alpha}$ is one-dimensional.

Proposition 2.5. (i) Let $V$ be an isometry on a separable infinite-dimensional Hilbert space $H$ and let $\alpha$ be the *-endomorphism of $B(H)$ defined by $\alpha(A)=$ $V A V^{*}$. Then $\alpha$ is a shift if and only if $V$ is either a pure isometry or $\bigcap_{r} V^{r} H$ is one-dimensional.

(ii) Let $\alpha, \beta$ be shifts on $B(H)$ defined by $\alpha(A)=V A V^{*}$ and $\beta(A)=$ $W A W^{*}$, where $V$ and $W$ are isometries on $H$. Then $\alpha$ is conjugate to $\beta$ if and only if $V$ is unitarily equivalent to $\lambda W$ for some $\lambda \in S^{1}$ i.e. if and only if $V$ and $W$ have the same Wold decomposition.

Proof. (i) By Proposition 2.4, the conditions on $V$ are necessary for $\alpha$ to be a shift. Conversely, if $V$ satisfies the given conditions, then $V$ can be assumed to be a direct sum of copies of the unilateral shift and (possibly) a unitary map on a 
one-dimensional subspace. Let $\left\{e_{i}: i \in \mathbb{N}\right\}$ be the orthonormal basis associated with one of the shift summands. If $T \in \alpha^{r}(B(H))$ then $T=V^{r} S V^{* r}$ for some $S \in B(H)$ and hence $T e_{i}=0$ for $1 \leq i \leq r$. It follows that, if $T \in \bigcap_{r} \alpha^{r}(B(H))$ then the restriction of $T$ to $\left(\bigcap_{r} V^{r} H\right)^{\perp}$ is 0 . The same argument applies to $T^{*}$ and hence $T$ leaves $\bigcap_{r} V^{r} H$ globally invariant. Therefore $\bigcap_{r} \alpha^{r}(B(H))=\mathbb{C} P$ where $P$ is the projection from $H$ onto the zero or one-dimensional space $\bigcap_{r} V^{r} H$.

(ii) This follows from Proposition 2.2.

The case in which $E_{\alpha}$ has dimension one is very special. For example, in contrast to the result of Proposition 2.5(i), if $E_{\alpha}$ has dimension greater than one, then each isometry $V$ in $E_{\alpha}$ is onto a space of infinite codimension and hence must be equivalent to an infinite direct sum of copies of the unilateral shift, possibly with an additional one-dimensional unitary summand.

Later in the paper an example will be produced to show that it may occur that $\alpha(A)=\sum V_{n} A V_{n}^{*}=\sum W_{n} A W_{n}^{*}$ where all the $V_{n}$ are pure and one of the $W_{n}$ is not. It will also be shown that the condition that all the $V_{n}$ are pure is not sufficient to ensure that $\alpha$ is a shift. Both these examples rely on the notion of a product shift, that will now be introduced.

\section{Product ShIFTS}

Let $K$ be a Hilbert space and, for each $i \in \mathbb{N}$, let $k_{i} \in K$ be a unit vector such that $\sum_{i}\left|1-\left\langle k_{i}, k_{i+1}\right\rangle\right|<\infty$. Recall that, as described in [3] or [6], the incomplete infinite tensor product $\otimes^{k_{i}} K$ is the Hilbert space inductive limit of the sequence $K \rightarrow K \otimes K \rightarrow K \otimes K \otimes K \cdots$, where the $i$ th map takes $h$ to $h \otimes$ $k_{i+1}$. Let $\phi_{i}$ be the natural map from the $i$-fold tensor product $K \otimes K \otimes \cdots \otimes K$ into $\otimes{ }^{k_{i}} K$ and let $\psi_{i}$ be the corresponding map from $K \otimes K \otimes \cdots \otimes K$ into $\otimes^{k_{i+1}} K$. Then, by [3, Proposition 1.1], for each $h$ in the $r$-fold tensor product $K \otimes K \otimes \cdots \otimes K$, the sequence $\phi_{r}(h), \phi_{r+1}\left(h \otimes k_{r+2}\right), \phi_{r+2}\left(h \otimes k_{r+2} \otimes k_{r+3}\right), \ldots$ has a limit in $\otimes^{k_{i}} K$ and, by [3, Proposition 1.3], the map taking $\psi_{r}(h)$ to this limit extends to an isomorphism $\theta$ from $\otimes^{k_{i+1}} K$ onto $\otimes^{k_{i}} K$. Informally, we can describe $\theta$ as taking $\otimes x_{i}$ (where $x_{i}=k_{i+1}$ for almost all $i$ ) to $\otimes x_{i}$ (interpreting the last expression as the limit of $\left.\phi_{1}\left(x_{1}\right), \phi_{2}\left(x_{1} \otimes x_{2}\right), \ldots\right)$.

Proposition 3.1. Let $P$ be a projection on $K$; $k_{i}$ be a sequence of unit vectors in $K$ with $\sum\left|1-\left\langle k_{1}, k_{i+1}\right\rangle\right|<\infty$; and $\theta$ be the isomorphism from $\otimes^{k_{i+1}} K$ onto $\otimes^{k_{i}} K$ taking $\otimes x_{i}$ (where $x_{i}=k_{i+1}$ for almost all $\left.i\right)$ to $\otimes x_{i}$. Then the map $\alpha_{k, P}$ defined on $B(H)=B\left(\otimes^{k_{i}} K\right)$ by $\alpha_{k, P}(T)=\theta(P \otimes T) \theta^{-1}$ is a shift.

Proof. The map $\alpha_{k, P}$ is clearly a *-endomorphism. In the unital case, $\alpha_{k, 1}^{r}(B(H))^{\prime}=B(K \otimes \cdots \otimes K) \otimes \mathbb{C l}$ (where the tensor product has $r$ factors) and hence $\bigcup_{r} \alpha_{k, 1}^{r}(B(H))^{\prime}$ is weakly dense in $B(H)$, from which it follows that $\alpha_{k, 1}$ is a shift. In the nonunital case let $q$ be the greatest lower bound 
of the projections $\alpha_{k, P}^{r}(1)$. If $q=0$ then, since $\alpha_{k, P}^{r}(1) T=T$ for each $T \in \bigcap_{r} \alpha_{k, P}^{r}(B(H)), T=q T=0$ for all such $T$ and hence $\alpha_{k, P}$ is a shift. If $q \neq 0$ then $q \alpha_{k, 1}^{r}(T) q=q \alpha_{k, P}^{r}(T) q$ for all $T$ because, as can be checked by induction, $\alpha_{k, P}^{r}(1) \alpha_{k, 1}^{r}(T) \alpha_{k, P}^{r}(1)=\alpha_{k, P}^{r}(T)$. Thus, since $\alpha_{k, P}(q)=q$, $\alpha_{k, P}^{r}(q B(H) q)=q \alpha_{k, P}^{r}(B(H)) q=q \alpha_{k, 1}^{r}(B(H)) q$ and so, when both algebras are restricted to $q H,\left[\alpha_{k, P}^{r}(q(B(H)) q)\right]^{\prime}=q \alpha_{k, 1}^{r}(B(H))^{\prime} q$. Hence, as in the unital case, $\bigcap_{r} \alpha_{k, P}^{r}(q B(H) q)=\mathbb{C} q$; however, $\bigcap_{r} \alpha_{k, P}^{r}(B(H)) \subseteq q B(H) q$ and, therefore, $\bigcap_{r} \alpha_{k, P}^{r}(B(H))=\mathbb{C} q$, as required.

If there exists a unitary $U$ from a Hilbert space $H$ onto an infinite tensor product $\otimes^{k_{i}} K$ with the properties above, then any *-endomorphism $\alpha$ of $B(H)$ of the form $\left(\operatorname{Ad} U^{*}\right) \alpha_{k, P}(\operatorname{Ad} U)$ will be called a product shift on $B(H)$.

Not every shift on $B(H)$ is a product as can be seen from Proposition 2.4. Any product shift $\alpha_{k, P}$ has $\alpha_{k, P}(1)$ of infinite codimension whereas if $\alpha$ is the shift corresponding to a single unilateral shift $V$ then $\alpha(1)$ has codimension 1 . Nevertheless the author is not aware of examples of nonproduct unital shifts on $B(H)$ : the following proposition shows that any sequence of isometries defining such a shift must be all pure.

Proposition 3.2. Let $\alpha$ be a unital shift on $B(H)$ defined by $\alpha(A)=\sum_{n} V_{n} A V_{n}^{*}$ where $V=V_{1}$ is not pure. Then $\alpha$ is a product shift.

Proof. Let $h$ be a unit vector in $\bigcap_{r} V^{r} H$ and note that, by Proposition 2.4, $\bigcap_{r} V^{r} H$ is one-dimensional. It follows that $V h=\lambda h$ for some $\lambda \in S^{1}$ and, replacing $V$ by $\bar{\lambda} V$, we can assume that $V h=h$.

Let $c_{00}$ denote the space of functions $f: \mathbb{N} \rightarrow \mathbb{N}$ such that $f(r)=1$ for all sufficiently large $r$ and, for each $f \in c_{00}$, let $b_{f}=\phi_{s}\left(V_{f(1)} \otimes \cdots \otimes V_{f(s)}\right) \in$ $\otimes^{V} E_{\alpha}$, where $\phi_{s}$ is the natural map from the $s$-fold tensor product $E_{\alpha} \otimes \cdots \otimes E_{\alpha}$ into $\otimes^{V} E_{\alpha}$ and where $s$ is chosen sufficiently large so that $f(r)=1$ for all $r \geq s$. Then, by [6, Lemma 4.1.4], $\left\{b_{f}: f \in c_{00}\right\}$ is an orthonormal basis for $\otimes^{V} E_{\alpha}$. Define $\psi: \otimes^{V} E_{\alpha} \rightarrow H$ extending $\psi\left(b_{f}\right)=V_{f(1)} \cdots V_{f(s)} h$ (which is well defined since $V h=h$ ). Then, for $t \geq s,\left\langle\psi\left(b_{f}\right), \psi\left(b_{g}\right)\right\rangle=$ $\left\langle V_{f(1)} \cdots V_{f(s)} h, V_{g(1)} \cdots V_{g(t)} h\right\rangle=\delta_{f(1) g(1)} \cdots \delta_{f(s) g(s)}\left\langle V_{g(t)}^{*} \cdots V_{g(s+1)}^{*} h, h\right\rangle=$ $\delta_{f g}\langle h, h\rangle=\delta_{f g}$ so that $\psi$ defines a unitary mapping from $\otimes^{V} E_{\alpha}$ onto a subspace $K$ of $H$.

For each $\mu, \nu$ with $|\mu| \geq 0$ and $|\nu|=r \geq 0, V_{\mu} V_{\nu}^{*} h=V_{\mu} V_{\nu}^{*} V^{r} h=$ $\delta_{\nu(1, \ldots, 1)} V_{\mu} h$, so that $K$ is equal to the closed linear span of the vectors $V_{\mu} V_{\nu}^{*} h$ with $|\mu| \geq 0$ and $|\nu| \geq 0$. By Lemma 2.3 (i) it follows that the projection onto $K$ belongs to $\bigcap_{r} \alpha^{r}(B(H))$ and hence, since $\alpha$ is a unital shift, $K=H$.

Note that

$$
\begin{aligned}
\left(\psi^{*} V_{n} \psi\right)\left(\phi_{r}\left(V_{f(1)} \otimes \cdots \otimes V_{f(r)}\right)\right) & =\psi^{*} V_{n} V_{f(1)} \cdots V_{f(r)} h \\
& =\phi_{r+1}\left(V_{n} \otimes V_{f(1)} \otimes \cdots \otimes V_{f(r)}\right),
\end{aligned}
$$


from which it follows that

$$
\left(\psi^{*} V_{n}^{*} \psi\right)\left(\phi_{r}\left(V_{f(1)} \otimes \cdots \otimes V_{f(s)}\right)\right)=\left\langle V_{f(1)}, V_{n}\right\rangle \phi_{r-1}\left(V_{f(2)} \otimes \cdots \otimes V_{f(r)}\right) ;
$$

hence

$$
\begin{aligned}
& \left(\operatorname{Ad} \psi^{*}\right) \alpha(\operatorname{Ad} \psi)\left(T_{1} \otimes T_{2} \otimes \cdots \otimes T_{s} \otimes 1\right) \\
& \quad=\sum_{n}\left(\psi^{*} V_{n} \psi\right)\left(T_{1} \otimes \cdots \otimes T_{s} \otimes 1\right)\left(\psi^{*} V_{n} \psi\right)^{*}={ }^{1} \otimes T_{1} \otimes \cdots \otimes T_{s} \otimes 1
\end{aligned}
$$

and thus that $\alpha$ is a product shift.

It will now be shown how product shifts can be defined to illustrate the comments made after Proposition 2.5.

Example 3.3. Let $K=\mathbb{C}^{2} ; k_{i}=(1,0)$ for each $i$; and the isometries $V_{1}, V_{2}$, $W_{1}, W_{2}$ be defined on $H=\otimes^{k_{i}} K$ by

$$
\begin{aligned}
V_{1}(h) & =(1 / \sqrt{2}, 1 / \sqrt{2}) \otimes h, & V_{2}(h) & =(1 / \sqrt{2},-1 / \sqrt{2}) \otimes h, \\
W_{1}(h) & =(1,0) \otimes h, & W_{2}(h) & =(0,1) \otimes h .
\end{aligned}
$$

Then $\alpha_{k, 1}(A)=V_{1} A V_{1}^{*}+V_{2} A V_{2}^{*}=W_{1} A W_{1}^{*}+W_{2} A W_{2}^{*}$ for each $A \in B(H)$. However, both $V_{1}$ and $V_{2}$ are pure isometries $\left(\right.$ as is $\left.W_{2}\right)$ but $\bigcap_{n} W_{1}^{n} H=\mathbb{C} k$, where $k=\otimes(1,0)$.

Example 3.4. Let $H, V_{1}, V_{2}$, and $\alpha_{k, 1}$ be as defined in Example 3.3 and let $\mathscr{H}$ be any Hilbert space. Then $\alpha=\alpha_{k, 1} \otimes$ id on $B(H) \otimes B(\mathscr{H})$ is not a shift; however,

$$
\alpha(A)=\left(V_{1} \otimes 1\right) A\left(V_{1}^{*} \otimes 1\right)+\left(V_{2} \otimes 1\right) A\left(V_{2}^{*} \otimes 1\right)
$$

for each $A$, and both $V_{1} \otimes 1$ and $V_{2} \otimes 1$ are pure isometries. Hence the converse of Proposition 2.4 is false.

The next main result gives a criterion for two unital product shifts to be conjugate. The proof is based on the following lemma:

Lemma 3.5. Let $W: \otimes^{k_{i}} K \rightarrow \bigotimes^{h_{i}} K$ be a unitary for which (Ad $\left.W\right) \alpha_{k, 1}\left(\operatorname{Ad} W^{*}\right)$ $=\alpha_{h, 1}$. Then there exists a unitary $V$ on $K$ such that, for each $r \geq 1$ and each $T_{1}, \ldots, T_{r} \in B(K),(\operatorname{Ad} W)\left(T_{1} \otimes \cdots \otimes T_{r} \otimes 1\right)=\left(V T_{1} V^{*}\right) \otimes\left(V T_{2} V^{*}\right) \otimes$ $\cdots \otimes\left(V T_{r} V^{*}\right) \otimes 1$.

Proof. For notational convenience, let $H_{h}=\bigotimes^{h_{i}} K$ and $H_{k}=\bigotimes^{k_{i}} K$. Then $\alpha_{h, 1}\left(B\left(H_{h}\right)\right)^{\prime}=\operatorname{Ad}(W)\left[\alpha_{k, 1}\left(B\left(H_{k}\right)\right)^{\prime}\right]$ and hence, for each $T \in B(K)$,

$(\operatorname{Ad} W)(T \otimes 1 \otimes 1 \otimes \cdots)=V T V^{*} \otimes 1 \otimes 1 \otimes \cdots$

for some unitary $V$ on $K$. Then, for each $r \geq 0$,

$$
\begin{aligned}
(\operatorname{Ad} W) \alpha_{k, 1}^{r}(T \otimes 1 \otimes 1 \otimes \cdots) & =\alpha_{h, 1}^{r}(\operatorname{Ad} W)(T \otimes 1 \otimes 1 \otimes \cdots) \\
& =\alpha_{h, 1}^{r}\left(V T V^{*} \otimes 1 \otimes 1 \otimes \cdots\right) .
\end{aligned}
$$


Hence, for each $r \geq 0,(\operatorname{Ad} W)\left(T_{1} \otimes \cdots \otimes T_{r} \otimes 1\right)=\left(V T_{1} V^{*}\right) \otimes\left(V T_{2} V^{*}\right) \otimes \cdots \otimes$ $\left(V T_{r} V^{*}\right) \otimes 1$.

Theorem 3.6. The product shifts $\alpha_{k, 1}$ and $\alpha_{h, 1}$ are conjugate if and only if there exists a unitary map $V$ on $K$ with $\sum|1-|\left\langle V k_{i}, h_{i}\right\rangle||<\infty$.

Proof. If $\sum|1-|\left\langle V k_{i}, h_{i}\right\rangle||<\infty$ then, by [3, Proposition 1.3], the map taking $\otimes x_{i}$ (where $x_{i}=k_{i}$ for almost all $i$ ) to $\otimes x_{i}$ extends to an isomorphism from $\otimes^{k_{i}} K$ onto $\otimes^{\alpha_{i} V^{*} h_{i}} K$, where $\alpha_{i}$ is the complex number of unit modulus such that $\left|\left\langle k_{i}, V^{*} h_{i}\right\rangle\right|=\left\langle k_{i}, \alpha_{i} V^{*} h_{i}\right\rangle$. However there is an isomorphism from $\otimes^{\alpha_{i} V^{*} h_{i}} K$ onto $\otimes^{h_{i}} K$ taking $\otimes x_{i}$ to $\otimes \bar{\alpha}_{i} V x_{i}$ and hence there is an isomorphism $W$ from $\otimes^{k_{i}} K$ onto $\otimes^{h_{i}} K$ taking $\otimes x_{i}$ (where $x_{i}=k_{i}$ for almost all $i)$ to $\otimes \bar{\alpha}_{i} V x_{i}$. Then, for $T_{1}, \ldots, T_{r} \in B(K)$,

$$
(\operatorname{Ad} W) \alpha_{k, 1}\left(\operatorname{Ad} W^{*}\right)\left(T_{1} \otimes \cdots \otimes T_{r} \otimes 1\right)=\alpha_{h, 1}\left(T_{1} \otimes \cdots \otimes T_{r} \otimes 1\right),
$$

from which it follows, by linearity and continuity that $\alpha_{k, 1}$ is conjugate to $\alpha_{h, 1}$.

Conversely, if $\alpha_{k, 1}$ is conjugate to $\alpha_{h, 1}$ then, by Lemma 3.5, there exists an isomorphism $W$ from $\otimes^{k_{i}} K$ onto $\otimes^{h_{i}} K$ and a unitary $V$ on $K$ such that $(\operatorname{Ad} W)\left(T_{1} \otimes \cdots \otimes T_{r} \otimes 1\right)=\left(V T_{1} V^{*}\right) \otimes \cdots \otimes\left(V T_{r} V^{*}\right) \otimes 1$ for all $T_{1}, \ldots, T_{r} \in B(K)$. In particular, this equation holds when $T_{i}$ is the onedimensional projection onto the subspace spanned by $k_{i}$. In this case, the decreasing sequence of projections $T_{1} \otimes \cdots \otimes T_{r} \otimes 1$ is weakly convergent as $r \rightarrow \infty$; by considering the effect of $T_{1} \otimes \cdots \otimes T_{r} \otimes 1$ on an orthonormal basis $\left\{e_{1 i_{1}} \otimes e_{2 i_{2}} \otimes \cdots: i_{1}, i_{2}, \ldots \in \mathbb{N}\right\}$ (where $e_{r 1}=k_{r}$ for each $r$ and $i_{k}=1$ for all sufficiently large $k$ ) it can be seen that the weak limit is the one-dimensional projection onto the subspace spanned by $k=\otimes k_{i}$. Hence the projections $\left(V T_{1} V^{*}\right) \otimes \cdots \otimes\left(V T_{r} V^{*}\right) \otimes 1$ are weakly convergent to a one-dimensional projection in $\otimes^{h_{i}} K$; let $x$ be a unit vector in the corresponding one-dimensional subspace. If $\phi_{r}$ denotes the natural map from the $r$-fold tensor product $K \otimes \cdots \otimes K$ into $\otimes^{h_{i}} K$ then there exists $s \in \mathbb{N}$ and a unit vector $x_{s}$ in $\phi_{s}(K \otimes \cdots \otimes K)$ such that $\left\|x-x_{s}\right\|<\frac{1}{2}$. Then, for each $r$,

$$
\begin{aligned}
& \left\|\left[\left(V T_{1} V^{*}\right) \otimes \cdots \otimes\left(V T_{r} V^{*}\right) \otimes 1\right] x_{s}\right\| \\
& \quad \geq\|x\|-\left\|x-\left[\left(V T_{1} V^{*}\right) \otimes \cdots \otimes\left(V T_{r} V^{*}\right) \otimes 1\right] x_{s}\right\|>\frac{1}{2} .
\end{aligned}
$$

It follows that $\prod_{i=s+1}^{r}\left|\left\langle k_{i}, V^{*} h_{i}\right\rangle\right| \nrightarrow 0$ as $r \rightarrow \infty$ and hence, by Lemma 2.4.1 (II) of [6], $\sum|1-|\left\langle k_{i}, V^{*} h_{i}\right\rangle||<\infty$, as required.

Theorem 3.6 can be used to produce examples of nonconjugate but outerconjugate unital shifts. Let $\log ^{k}$ denote the $k$-fold composite $\log \circ \cdots \circ \log$ and, for each $r \in \mathbb{N}$, define a sequence $f_{r}$ by

$$
f_{r}(n)= \begin{cases}1 & \text { if } \log ^{r}(n) \leq 0, \\ 1 /\left(n \log (n) \log ^{2}(n) \cdots \log ^{r}(n)\right) & \text { if } \log ^{r}(n)>0 .\end{cases}
$$


Let $K$ be a separable Hilbert space (of dimension at least 2) with an orthonormal basis $\left\{e_{i}\right\}$ and, for each $n \in \mathbb{N}$, define $k_{r, n} \in K$ by $k_{r, n}=$ $\left(1-f_{r}(n)\right) e_{1}+\left[f_{r}(n)\left(2-f_{r}(n)\right)\right]^{1 / 2} e_{2}$.

Lemma 3.7. (i) $\left\|k_{r, n}\right\|=1$ for each $r$ and $n$.

(ii) $\sum_{n}\left|1-\left\langle k_{r, n}, k_{r, n+1}\right\rangle\right|<\infty$ for each $r$.

Proof. Claim (i) is easily verified. To check claim (ii), note that

$$
0 \leq\left\langle k_{r, n}, k_{r, n+1}\right\rangle \leq\left\|k_{r, n}\right\|\left\|k_{r, n+1}\right\|=1
$$

and hence that

$$
\begin{aligned}
0 \leq & \left|1-\left\langle k_{r, n}, k_{r, n+1}\right\rangle\right| \\
= & f_{r}(n)+f_{r}(n+1)-f_{r}(n) f_{r}(n+1) \\
& -\left[f_{r}(n) f_{r}(n+1)\left(2-f_{r}(n)\right)\left(2-f_{r}(n+1)\right)\right]^{1 / 2} \\
\leq & 2 f_{r}(n)-f_{r}(n) f_{r}(n+1)-f_{r}(n+1)\left(2-f_{r}(n)\right) \\
= & 2\left(f_{r}(n)-f_{r}(n+1)\right) .
\end{aligned}
$$

However $\sum_{n} 2\left(f_{r}(n)-f_{r}(n+1)\right)$ is convergent, from which the result follows.

Proposition 3.8. For each $r \in \mathbb{N}$, let $\alpha_{r}$ be the product shift $\alpha_{k_{r}, 1}$ on $\bigotimes_{n=1}^{\infty} k_{r, n} K$, where $k_{r, n}$ is defined above. Then, if $r \neq s, \alpha_{r}$ is outer conjugate but not conjugate to $\alpha_{s}$.

Proof. For definiteness suppose that $r>s$. By construction $\alpha_{r}$ and $\alpha_{s}$ have the same multiplicity $\operatorname{dim}(K)$ and hence are outer conjugate by [7, Theorem 2.4]. If they are conjugate then, by Theorem 3.6, there exists a unitary $V$ on $K$ such that $\sum_{n}|1-|\left\langle V k_{r, n}, k_{s, n}\right\rangle||<\infty$. It follows that $\left|\left\langle V k_{r, n}, k_{s, n}\right\rangle\right| \rightarrow 1$ as $n \rightarrow \infty$ and hence that $\left|\left\langle V e_{1}, e_{1}\right\rangle\right|=1$, so that $V e_{1}=\alpha e_{1}$ for some $\alpha \in S^{1}$. Hence,

$$
\begin{aligned}
\left|\left\langle V k_{r, n}, k_{s, n}\right\rangle\right|= & \mid\left(1-f_{r}(n)\right)\left(1-f_{s}(n)\right)\left\langle\alpha e_{1}, e_{1}\right\rangle \\
& +\left[f_{r}(n) f_{s}(n)\left(2-f_{r}(n)\right)\left(2-f_{s}(n)\right)\right]^{1 / 2}\left\langle V e_{2}, e_{2}\right\rangle \mid \\
\leq & \left(1-f_{r}(n)\right)\left(1-f_{s}(n)\right)+2 f_{s}(n)^{1 / 2} f_{r}(n)^{1 / 2} \\
= & 1-f_{s}(n)\left[1-f_{r}(n)+f_{r}(n) f_{s}(n)^{-1}-2 f_{r}(n)^{1 / 2} f_{s}(n)^{-1 / 2}\right] .
\end{aligned}
$$

However, for all sufficiently large $n, f_{r}(n) f_{s}(n)^{-1}=1 /\left(\log ^{s+1}(n) \cdots \log ^{r}(n)\right) \rightarrow$ 0 as $n \rightarrow \infty$ and therefore, for all sufficiently large $n,\left|\left\langle V k_{r, n}, k_{s, n}\right\rangle\right| \leq$ $1-f_{s}(n) / 2$ and therefore, $\sum|1-|\left\langle V k_{r, n}, k_{s, n}\right\rangle||$ is not convergent, giving a contradiction.

\section{REFERENCES}

1. R. Archbold, On the 'flip-flop' automorphism of $C^{*}\left(S_{1}, S_{2}\right)$, Quart. J. Math. Oxford (2), 30 (1979), 129-132.

2. W. Arveson, Continuous analogues of Fock space, Mem. Amer. Math. Soc., no. 409, Amer. Math. Soc., Providence, RI, 1989. 
3. A. Guichardet, Produits tensoriels infinis et représentations des relations d'anticommutation, Ann. Sci. Ecole Norm. Sup. (3) 83 (1966), 1-52.

4. P. R. Halmos, A Hilbert space problem book, Springer-Verlag, Berlin and New York, 1974.

5. H. Hanche-Olsen and E. Størmer, Jordan operator algebras, Pitman, 1984.

6. J. von Neumann, On infinite direct products, Compositio Math. 6 (1938), 1-77.

7. R. T. Powers, An index theory for semigroups of *endomorphisms of $B(H)$ and type $\mathrm{II}_{1}$ factors, Canad. J. Math. 40 (1988), 86-114.

Department of Mathematics, La Trobe University, Bundoora, Victoria 3083, AusTRALIA 